\title{
Triggering Threshold Spacecraft Charging with Changes in Electron Emission from Materials
}

\author{
J. R. Dennison ${ }^{1}$ R. C. Hoffmann ${ }^{2}$ and J. Abbott ${ }^{3}$ \\ Physics Department, Utah State University, Logan, UT, USA 84322-4415
}

\begin{abstract}
[Abstract] Modest changes in spacecraft charging conditions can lead to abrupt changes in the spacecraft equilibrium, from small positive potentials to large negative potentials relative to the space plasma; this phenomenon is referred to as threshold charging. It is well known that temporal changes of the space plasma environment (electron plasma temperature or density) can cause threshold charging. Threshold charging can also result from by temporal changes in the juxtaposition of the spacecraft to the environment, including spacecraft orbit, orientation, and geometry. This study focuses on the effects of possible changes in electron emission properties of representative spacecraft materials. It is found that for electron-induced emission, the possible threshold scenarios are very rich, since this type of electron emission can cause either positive or negative charging. Alternately, modification of photon- or ion-induced electron emission is found to induce threshold charging only in certain favorable cases. Changes of emission properties discussed include modifications due to: contamination, degradation and roughening of surfaces and layered materials; biasing and charge accumulation; bandstructure occupation and density of states caused by heat, optical or particle radiation; optical reflectivity and absorptivity; and inaccuracies and errors in measurements and parameterization of materials properties. An established method is used here to quantitatively gauge the relative extent to which these various changes in electron emission alter a spacecraft's charging behavior and possibly lead to threshold charging. The absolute charging behavior of a hypothetical flat, twodimensional satellite panel of a single material (either polycrystalline conductor Au or the polymeric polyimide Kapton ${ }^{\mathrm{TM}} \mathrm{H}$ ) is modeled as it undergoes modification and concomitant changes in spacecraft charging in three representative geosynchronous orbit environments, from full sunlight to full shade (eclipse) are considered.
\end{abstract}

\section{Nomenclature}

\begin{tabular}{|c|c|c|}
\hline$A$ & $=$ & emission surface area \\
\hline$A_{n}$ & $=$ & optical absorptivity at normal incidence \\
\hline$b_{1}, b_{2}$ & $=$ & stopping power power-law magnitudes for NASCAP secondary electron yield fit \\
\hline$E_{e}, E_{\text {Ion }}, E_{P h}$ & $=1$ & incident electron, ion and photon energies \\
\hline$E_{1}, E_{2}$ & $=1$ & first and second crossover energies \\
\hline$E_{\max }^{\sigma}, E_{\max }^{\delta}$ & $=$ & energy at maximum total and secondary electron yields \\
\hline$J_{\text {net }}, J_{\text {incident }}, J_{e n}$ & mitted ${ }^{3}$ & $=$ net, incident and emitted current density \\
\hline$k_{B}$ & $=1$ & Boltzmann's constant \\
\hline$m_{e}$ & $=1$ & mass of the electron \\
\hline$N_{e}, N_{\text {Ion }}, N_{P h}$ & $=$ & incident electron, ion and photon fluxes, in particles per unit area and time \\
\hline$N^{e m}{ }_{e}, N^{e m}{ }_{I o n}, N^{e n}$ & $m{ }^{m} \mathrm{Ph}$ & $=$ electron-, ion-, and photon-induced emitted electron fluxes, in particles per unit area and tin \\
\hline$N_{e}, N_{\text {Ion }}, \hat{N}_{P h}$ & & incident electron, ion and photon differential fluxes, in particles per unit area, time and energy \\
\hline$n_{1}, n_{2}$ & $=$ & stopping power power-law exponents for NASCAP secondary electron yield fit \\
\hline$n_{e}, n_{\text {Ion }}$ & $=$ & electron and ion equilibrium plasma particle density, in number of particles per unit volume \\
\hline$q_{e}, q_{I o n}, q_{P h}$ & $=$ & magnitudes of electron, ion and photon charge per particle \\
\hline$Q_{\text {net }}$ & $=$ & $t$ accumulated charge \\
\hline
\end{tabular}

${ }^{1}$ Professor of Physics, Materials Physics Group, UMC 4415, JR.Dennison@usu.edu, Member.

${ }^{2}$ Graduate Student, Materials Physics Group, UMC 4415.

${ }^{3}$ Undergraduate Student, Materials Physics Group, UMC 4415. 


$\begin{array}{ll}R_{n} & =\text { optical reflectivity at normal incidence } \\ T_{e}, T_{I o n} & =\text { effective electron or ion temperature of Maxwellian plasma distribution } \\ T_{C r} & =\text { critical effective electron temperature at neutral yield } \\ \mathrm{T}_{n} & =\text { optical transmission at normal incidence } \\ Z_{e f f} & =\text { effective atomic number for NASCAP backscattered electron yield fit } \\ \delta & =\text { secondary electron yield } \\ \delta_{\max } & =\text { maximum secondary electron yield } \\ \overline{\langle\delta+\eta\rangle} & =\text { flux-weighted average electron-induced electron yield } \\ \Delta t & =\text { time interval } \\ \eta & =\text { backscattered electron yield } \\ \varphi & =\text { incidence angle relative to surface normal } \\ \sigma_{\text {Tot }} & =\text { total electron yield } \\ \sigma_{e}, \sigma_{\text {Ion }}, \sigma_{P h} & =\text { total electron-induced, ion-induced and photon-induced electron yields } \\ \overline{\left\langle\sigma_{I o n}\right\rangle}, \overline{\left\langle\sigma_{P h}\right\rangle} & =\text { flux-weighted average ion- and photon-induced electron yields } \\ \overline{\left\langle\sigma_{I o n}\right\rangle}, \overline{\left\langle\sigma_{P h}\right\rangle} & =\text { scaled, flux-weighted average ion- and photon-induced electron yields } \\ \sigma_{\max } & =\text { maximum total electron yield }\end{array}$

\section{Introduction}

TNERACTIONS of spacecrafts with the space environment can result in charge accumulation, a process referred Ito as spacecraft charging. Spacecraft can accumulate either positive or negative charge relative to the ambient plasma environment, depending on the specific circumstances. However, most common conditions limit positive charging to $\sim 10^{1} \mathrm{eV}$, while negative charging can often attain much higher potentials up to $10^{3}$ to $10^{4} \mathrm{eV}$. Ultimately, this asymmetry in equilibrium charge is a consequence of the higher mobility of the negative charge carriers (electrons), both within spacecraft materials and in the space plasma.

Charging is known to produce many of the spacecraft system anomalies, electron upsets, damage to components, power system debilities, and even complete satellite failures attributed to interactions with the natural space environment. ${ }^{1-3}$ Perhaps the most dangerous charging scenario is termed threshold charging, in which there is a rapid change between low-level positive charging and high-level negative charging that results from modest changes in the spacecraft charging conditions. To model such charging, the codes require information about: (i) the chargeinducing environment, including electron, ion, and photon fluxes over the full range of the energy spectrum; (ii) the juxtaposition of the spacecraft to the environment, including orbit, spacecraft orientation, and geometry; and (iii) the electronic properties of spacecraft materials and material response to the incident fluxes, including charged particle emission and resistivity. ${ }^{3-7}$

The purpose of the present study is to investigate the possible changes that can trigger threshold charging and to determine in a quantitative way the relative extent to which these changes might alter a spacecraft's charging behavior under general circumstances. We focus in particular on the effects of changes in electron emission from representative spacecraft materials. These changes are discussed in terms of a general framework for threshold charging, based on simple consideration of charge conservation at spacecraft equilibrium. The measured electron emission properties of two materials used in this study to illustrate various threshold charging effects are shown in Figs. 1, 2 and 3. These materials are high purity polycrystalline $\mathrm{Au}$ and thin film polymeric polyimide Kapton ${ }^{\mathrm{TM}} \mathrm{H}$.

\section{Causes of Threshold Charging}

The general behavior of threshold charging can be readily understood for simple scenarios in terms of charge conservation or a current balance equation. We limit our considerations here to surfaces of a single, homogenous material and to uniform, time-independent incident fluxes. We require knowledge of the energy distribution of the incident particles in the plasma environment (see Fig. 4), the electron yields as functions of incident energy (see Figs. 1(a) and 2(a)), the energy distribution of emitted electrons (see Figs. 1, 2 and 3), and the effects of biasing or charging on electron emission. ${ }^{19-21}$

The net accumulated charge is the difference between the time integral of the incident and emitted currents; that is, $Q_{\text {net }}$ accumulated on a surface area, $A$, in a short time interval, $\Delta t$, is given in terms of the net incident electron, ion and photon particle fluxes $\left(N_{e}, N_{\text {Ion }}\right.$ and $N_{P h}$, respectively, in number of incident particles per unit area and time) and the fluxes of emitted electrons due to them (superscripted $N^{e m}$ values; see Figs. 2(b) and 3(b)) as 


$$
\begin{aligned}
& J_{\text {net }}=J_{\text {Incident }}-J_{\text {Emitted }} \\
& Q_{\text {net }} / A \cdot \Delta t=\left[-q_{e} N_{e}+q_{\text {Ion }} N_{\text {Ion }}+q_{P h} N_{P h}\right]-\left(-q_{e}\right) \cdot\left[N_{e}^{e m}+N_{\text {Ion }}^{e m}+N_{P h}^{e m}\right]
\end{aligned}
$$

Here $q_{e}, q_{I o n}$ (assumed hereafter equal to $q_{e}$ for singly ionized atoms), and $q_{P h} \equiv 0$ are the magnitude of the charge on electrons, ions and photons, respectively. We assume that no appreciable numbers of ions or photons are emitted from the material, except where specifically noted. We also assume no charge redistribution within the material. Note that it is only the first term on the right hand side of Eq. (1), from absorption of incident electrons, that can contribute negative charge to the charge balance, since photons contribute no charge and incident ions and all emitted electrons contribute positive charge.

As the material comes to equilibrium with the plasma environment at charge $Q_{n e}$, incident current equals emitted current and the left-hand side of Eq. (1) asymptotically approaches zero. For this to occur, the initial (uncharged) electron yields are modified as charge accumulates. Positively biased surfaces recaptures sufficient low energy emitted electrons to establish an equilibrium between incident and emitted charge currents. Because the energy distributions of emitted electrons are peaked at $\sim 2-5 \mathrm{eV}$ (Figures 2(c) and 3(b) show representative secondary electron emission spectra; the emission spectra from other incident particles are quite similar irrespective of the incident energy source.), in most cases a positive equilibrium potential of $<10 \mathrm{eV}$ is sufficient to attain equilibrium. ${ }^{19}$ In sunlight, photoelectron emission is usually dominant, causing an accumulation of positive charge on the surface. In eclipse, there is no photoelectron emission; however, for most clean metal surfaces there is still sufficient net electron- and ion-induced electron emission to maintain a positive bias. ${ }^{22}$

If, on the other hand, there are more electron impinging on the surface than leaving (that is, if the first term of Eq. (1) dominates), this leads to a net negative charging. Low energy electrons are no longer recaptured by the surface, but rather are repelled by the negatively biased surface. Since negative bias does not significantly affect electron or photon yields at lower potentials, ${ }^{19}$ charge can accumulate rapidly, leading to an abrupt increase in negative charge accumulation. ( $\sigma_{\text {Ion }}$ is affected by negative charging

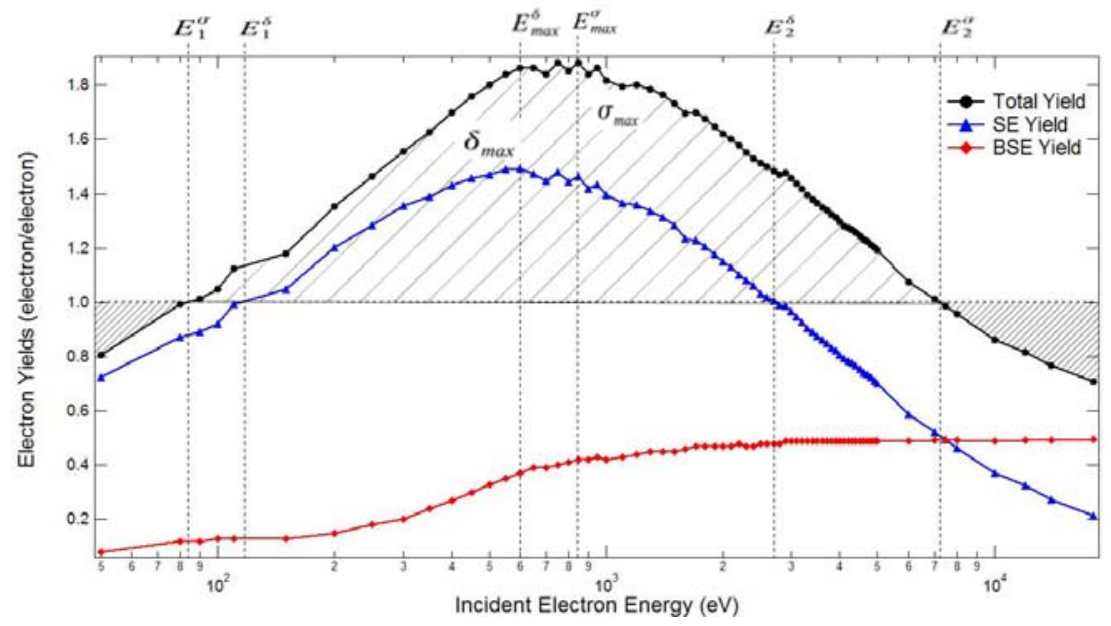

(a)

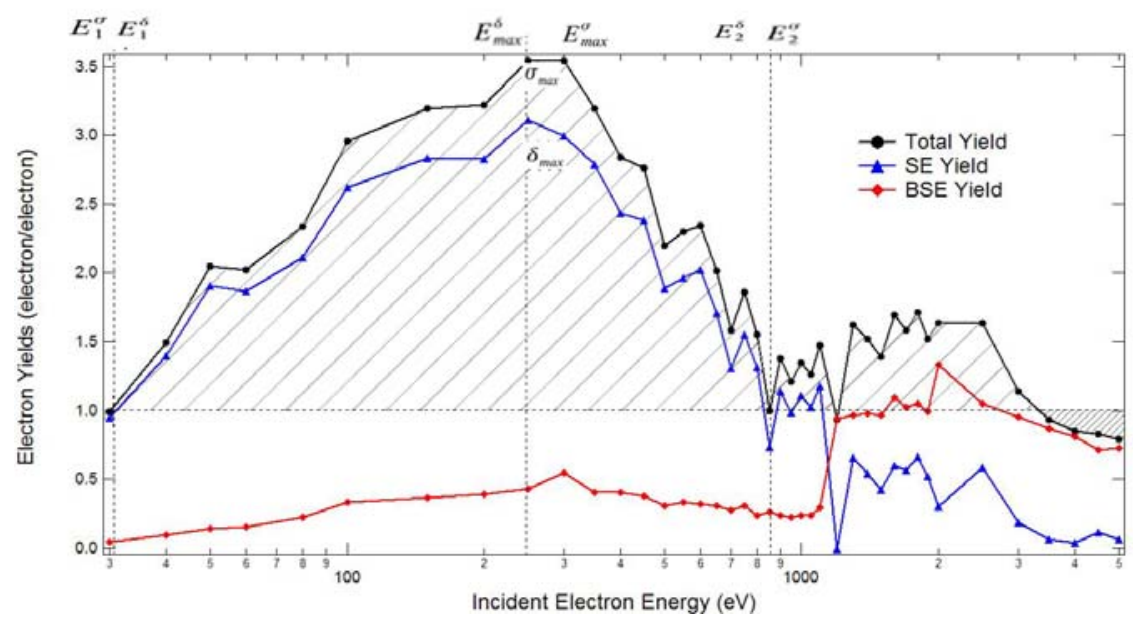

(b)

Figure 1. Total, secondary and backscattered electron-induced electron yield curves as a function of incident energy. ${ }^{8}$ Note the logarithmic energy scale. Also shown are the maximum total (secondary) yield, $\sigma_{\max },\left(\delta_{\max }\right)$ at energy $E_{\max }^{\sigma}\left(E_{\max }^{\delta}\right)$ and crossover energies $E_{1}$ and $E_{2}$ at which the total yield equals unity. Electrons above (below) $\sigma=1$ that contribute to positive (negative) charging are shown in dark (light) shading. (a) Polycrystalline high purity Au. (b) Kapton ${ }^{T M} H$ polyimide taken with a charge fluence of $\sim 120 \mathrm{fC} / \mathrm{pulse}(2.5$ fC.mm ${ }^{2}$-pulse). 


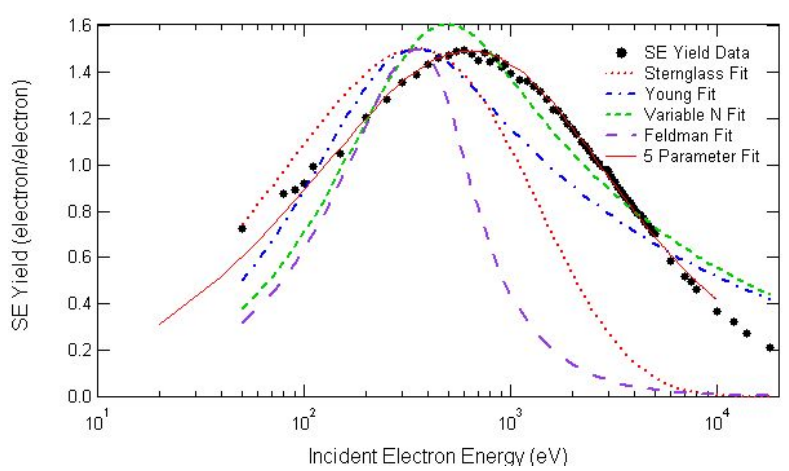

(a)

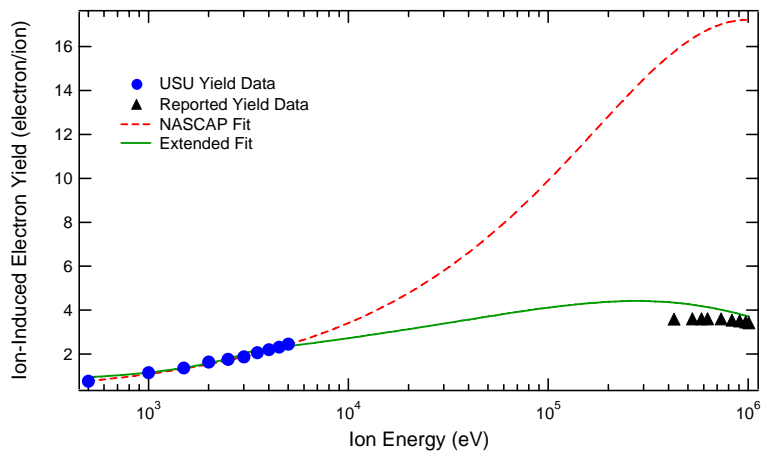

(c)

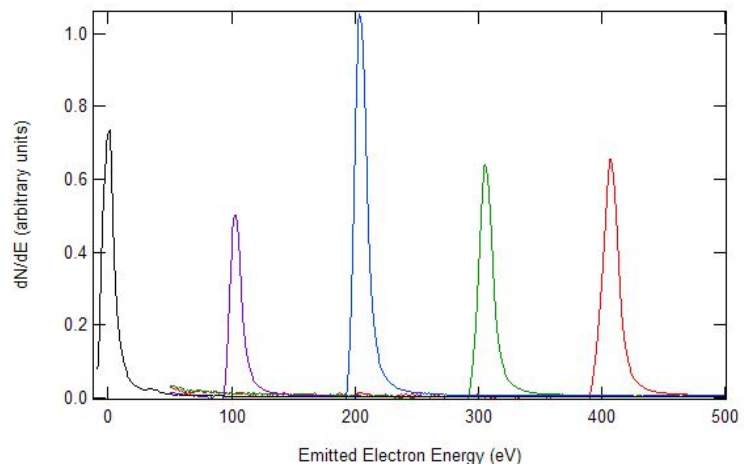

(b)

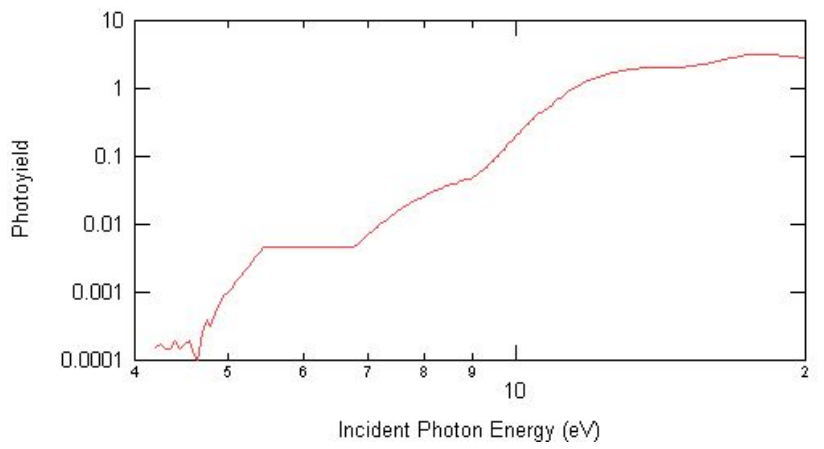

(d)

Figure 2. Electron emission data for polycrystalline Au. (a) Secondary electron yield as a function of incident energy. Note the logarithmic energy scale. Fits to the data are based on the Feldman, Young, variable n, NASCAP 5 parameter and Sternglass models, respectively. Fitting functions are described in. ${ }^{7-11}$ Fitting parameters are listed in Table I. (b) Electron emission spectra as a function of emission energy. Curves peaked from left to right are for samples biased to $0 \mathrm{~V},-100 \mathrm{~V},-200 \mathrm{~V},-300 \mathrm{~V}$ and $-400 \mathrm{~V}$, respectively. ${ }^{12}$ (c) Ion-induced electron yield curves as a function of incident ion energy. ${ }^{8}$ Note the logarithmic energy scale. Data at higher energies are taken from Refs. 13 and 14. Fits are shown for a two parameter fit used in NASCAP ${ }^{10}$ and a six parameter extended fit. ${ }^{8,11}$ Fitting parameters are listed in Table I. (d) Photon-induced electron yield curves as a function of incident photon energy. ${ }^{8}$ The onset of photoemission is evident at a work function of $\sim 4.7 \mathrm{eV}$. Data at energies above are from Refs. 15 and 16. Note the logarithmic yield scale.

since ions are attracted to the negative bias surface. However, ions usually have a much smaller contribution to the total yield than that from electrons or photons.) It is only when the bias potential reaches sufficiently high values to significantly inhibit the number of primary electrons from the plasma reaching the surface that this rapid accumulation of negative charge is slowed. ${ }^{19}$

In terms of the secondary electron, backscattered electron, ion and photon yields $\left(\delta, \eta, \sigma_{I o n}, \sigma_{P h}\right.$, respectively), at charge equilibrium Eq. (1) implies

$$
0_{<}^{>}[\delta+\eta-1] \cdot N_{e}+\left(1+\sigma_{\text {Ion }}\right) \cdot N_{\text {Ion }}+\sigma_{P h} \cdot N_{P h}
$$

The secondary electron (SE) and backscattered electron (BSE) yields, $\delta$ and $\eta$, are the ratio of low $(<50 \mathrm{eV})$ and high energy electrons emitted per incident primary electron (PE), respectively. This assumes a uniform flux, independent of incident energy. More correctly, to reflect conservation of charge at equilibrium potential, Eq. (2) should integrate the products of energy dependant yields (see Fig. 1) and incident differential fluxes (see Fig. 4) over all appropriate incident energies: 


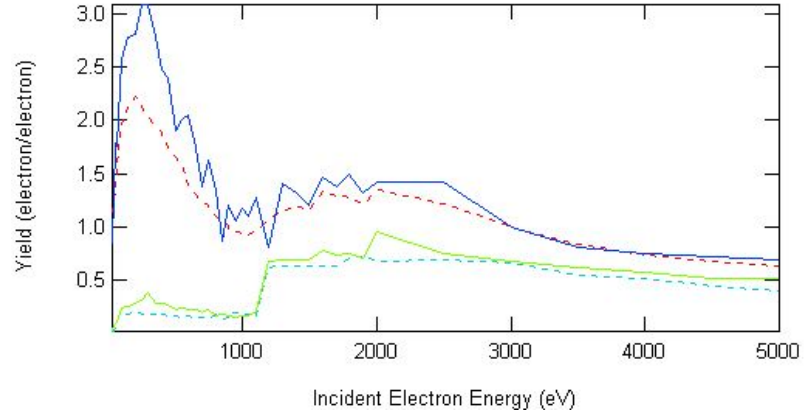

(a)

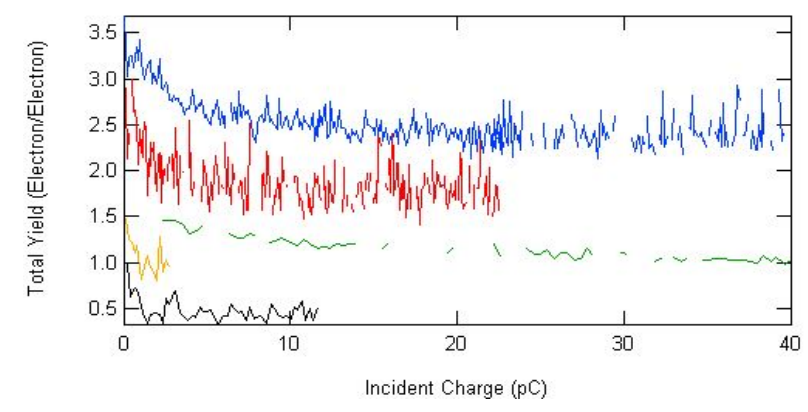

(c)

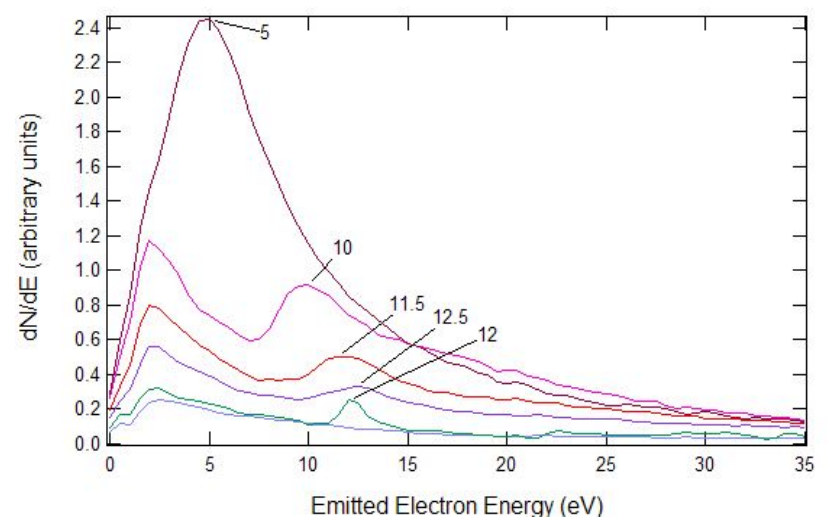

(b)

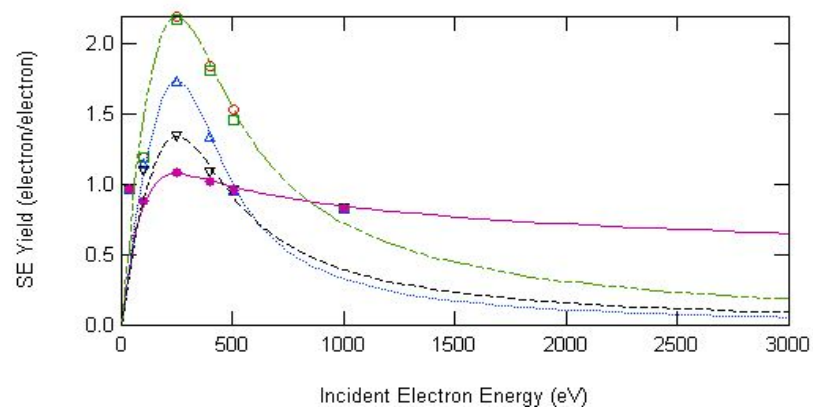

(d)

Figure 3. Effects of accumulated charge on the electron emission of polyimide Kapton ${ }^{\mathrm{TM}} \mathbf{H}$. (a) Total and backscattered electron yields as a function of incident energy. Solid and dashed curves are taken at fluences per pulse of $\sim 0.4 \mathrm{pC} / \mathrm{pulse}\left(\sim 80 \mathrm{pC} / \mathrm{mm}^{2}\right)$ and $3 \mathrm{pC} / \mathrm{pulse}\left(\sim 600 \mathrm{pC} / \mathrm{mm}^{2}\right)$, respectively. (b) Electron emission spectra as a function of emission energy. Curves from top to bottom were taken at incident energies of $-100 \mathrm{eV},-90 \mathrm{eV},-80$ $\mathrm{eV},-70 \mathrm{eV},-60 \mathrm{eV}$ and $-50 \mathrm{eV}$, respectively. ${ }^{12}$ Curves from top to bottom exhibit peaks at $5.0 \mathrm{eV}, 10.0 \mathrm{eV}, 11.5 \mathrm{eV}$, $12.5 \mathrm{eV}$ and $12 \mathrm{eV}$ corresponding to negative sample potentials of $-3.0 \mathrm{eV},-8.0 \mathrm{eV},-9.5 \mathrm{eV},-10.5 \mathrm{eV}$ and $-10.0 \mathrm{eV}$, respectively. The stationary peaks at $\sim 2 \mathrm{eV}$ are due to SE emission of the detector retarding grid. ${ }^{17}$ (c) Electron total yield decay curves as a function of incident charge density. ${ }^{18}$ Curves from top to bottom show yields at incident energies of $250 \mathrm{eV}, 100 \mathrm{eV}, 300 \mathrm{eV}, 400 \mathrm{eV}$ and $500 \mathrm{eV}$ with asymptotic limits to the yield at large charge fluence of 1.2, 1.0 1.0, 1.2 and 1.1, respectively. Curves have been offset vertically by values of $+1.0,+0.50 .0,-0.2$ and -0.5, from top to bottom respectively, to separate the data for clarity. (d) Composite total electron yield curves estimated from data from the total yield decay curves in (c). Curves, from top to bottom at $\delta_{\text {max }}$, are for total yields

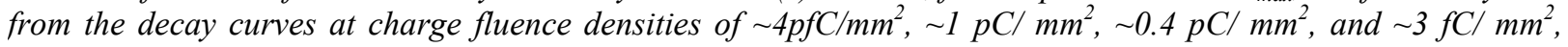
respectively.

$$
\int \dot{N}_{e}\left(E_{e}\right) \cdot d E_{e}>\int\left[\delta\left(E_{e}\right)+\eta\left(E_{e}\right)\right] \cdot \dot{N}_{e}\left(E_{e}\right) \cdot d E_{e}+\left\{\int\left[1+\sigma_{\text {Ion }}\left(E_{i}\right)\right] \cdot \dot{N}_{\text {Ion }}\left(E_{i}\right) \cdot d E_{i} \quad+\int \sigma_{P h}\left(E_{P h}\right) \cdot \dot{N}_{P h}\left(E_{P h}\right) \cdot d E_{P h}\right\}
$$

Dividing through by the total incident electron flux, $N_{e} \equiv \int N_{e}\left(E_{e}\right) \cdot d E_{e}$, Eq. (3) may be expressed in a more compact notation as

$$
1_{<}^{>} \overline{\langle\delta+\eta\rangle}+\left\{\left(N_{\text {Ion }} / N_{e}\right) \cdot \overline{\left\langle 1+\sigma_{\text {Ion }}\right\rangle}+\left(N_{P h}^{t} / N_{e}\right) \cdot \overline{\left\langle\sigma_{P h}\right\rangle}\right\} \equiv \overline{\langle\delta+\eta\rangle}+\left\{\overline{\left\langle 1+\sigma_{\text {Ion }}\right\rangle_{e}}+\overline{\left\langle\sigma_{P h}\right\rangle_{e}}\right\}
$$

The terms in Eqs. (3) and (4) are arranged such that those on the left-hand side contribute negative charge to the balance and those on the right contribute positive charge. In Eq. (4), the average electron-induced yield integrated over the full range of incident electron energies and weighted by the incident electron flux distribution, $N_{e}\left(E_{e}\right)$, is 
(a)

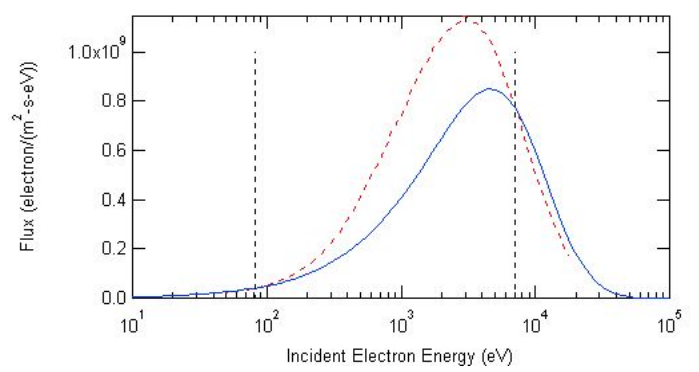

(c)

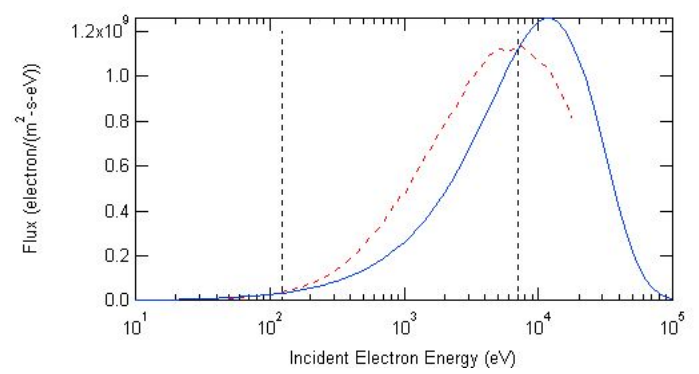

(e)

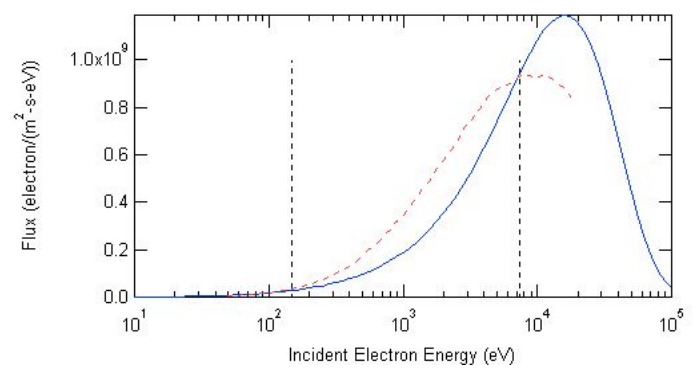

(b)

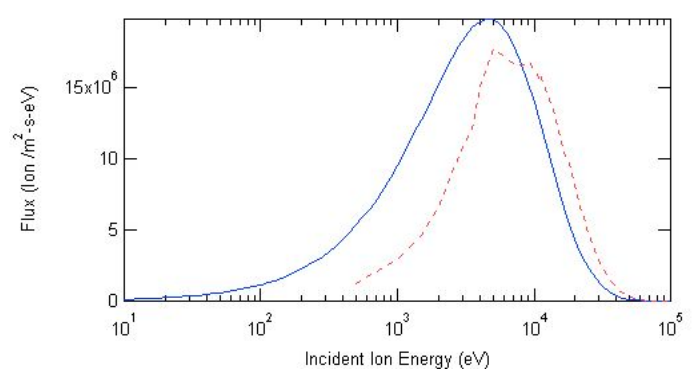

(d)

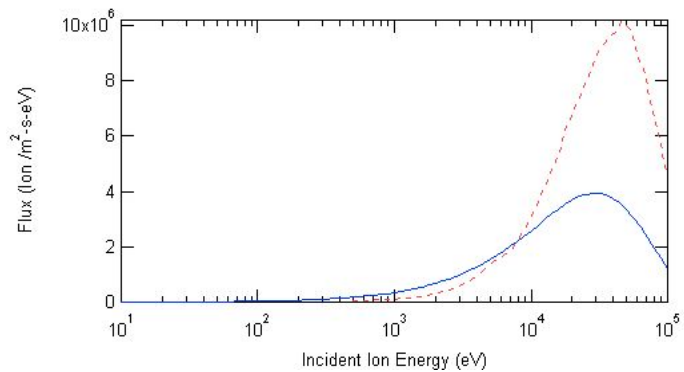

(f)

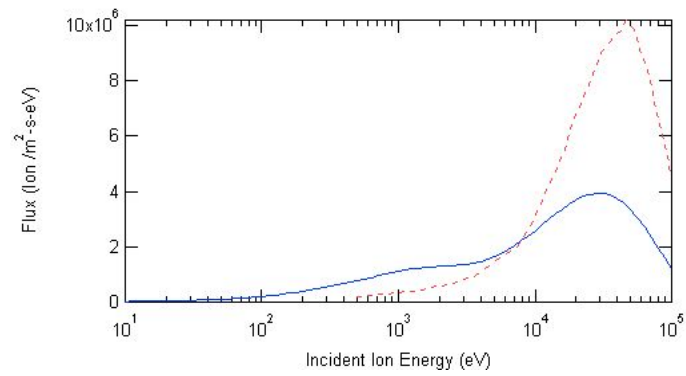

(g)

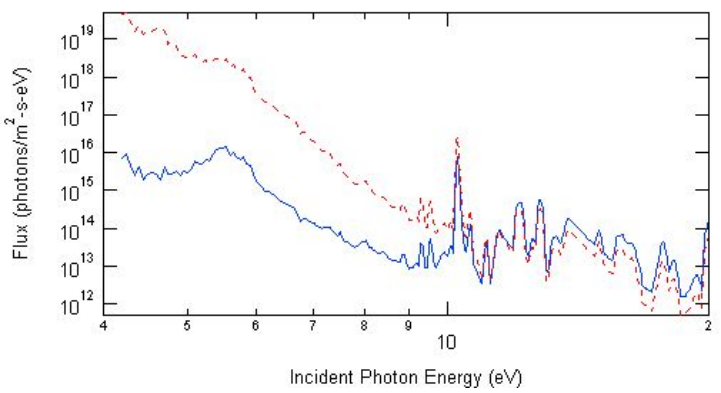

Figure 4. Energy dependant incident differential flux densities, $N(E)$, (blue solid lines) and the product of yield times differential flux densities, $\sigma(E) \cdot N(E)$, (blue dashed lines) as functions of incident energy. Yields used are for polycrystalline Au, as shown in Figs. (1) and (2). Note the logarithmic energy scales. Fluxes are shown for electrons (Figs. (a), (c) and (e)) and ions (Figs. (b), (d) and (f)) for three representative geosynchronous environments listed in NASCAP: ${ }^{10}$ (i) Figs. (a) and (b) for a "standard day" of 4 September 1997 (bi-Maxwellian electron distribution with $n_{1}=3.00 \cong 10^{5} \mathrm{~m}^{-3}, T_{1}=4000 \mathrm{eV}, n_{2}=2.00 \cong 10^{5} \mathrm{~m}^{-3}$, and $T_{2}=7000 \mathrm{eV}$ and Maxwellian ion distribution with $n_{1}=3.00 \cong 10^{5} \mathrm{~m}^{-3}$ and $T_{1}=4000 \mathrm{eV}$ ); (ii) Figs. (c) and (d) for a typical "worst case" environment $t^{23}$ (Maxwellian electron distribution with $n_{1}=1.12 \cong 10^{6} \mathrm{~m}^{-3}$ and $T_{1}=12000 \mathrm{eV}$ and Maxwellian ion distribution with $n_{1}=1.12 \cong 10^{6} \mathrm{~m}^{-3}$ and $T_{1}=12000 \mathrm{eV}$ ); and (iii) Figs. (e) and (f) for an extreme environment encountered by the Advanced Technology Satellite-6 (bi-Maxwellian electron distribution with $n_{1}=1.22 \cong 10^{6} \mathrm{~m}^{-3}$, $T_{1}=16000 \mathrm{eV}, \quad n_{2}=2.36 \cong 10^{5} \mathrm{~m}^{-3}$, and $T_{2}=29500 \mathrm{eV}$ and Maxwellian ion distribution with $n_{1}=1.22 \cong 10^{6} \mathrm{~m}^{-3}$ and $T_{1}=16000 \mathrm{eV}$ ). The two curves for the electron fluxes in Figs. (a), (c) and (e) cross at the cross over energies, $E_{1}$ (79 eV, $125 \mathrm{eV}$ and $150 \mathrm{eV}$, respectively) and $E_{2}(7079 \mathrm{eV}, 7075 \mathrm{eV}$ and $7500 \mathrm{eV}$, respectively), where the total electron yields are unity. (g) The standard solar (A0) photon differential flux density (or irradiance) ${ }^{24}$ and the product of the irradiance times photoyield are the same for all three environments. 


$$
\begin{aligned}
& \overline{\langle\delta+\eta\rangle} \equiv \int\left[\delta\left(E_{e}\right)+\eta\left(E_{e}\right)\right] \cdot \dot{N}_{e}\left(E_{e}\right) \cdot d E_{e} / \int \dot{N}_{e}\left(E_{e}\right) \cdot d E_{e} \\
& \overline{\left\langle 1+\sigma_{\text {Ion }}\right\rangle_{e}} \equiv\left(N_{\text {Ion }} / N_{e}\right) \cdot \overline{\left\langle 1+\sigma_{\text {Ion }}\right\rangle} \equiv\left(N_{\text {Ion }} / N_{e}\right) \cdot \int\left[1+\sigma_{\text {Ion }}\left(E_{\text {Ion }}\right)\right] \cdot \dot{N}_{\text {Ion }}\left(E_{\text {Ion }}\right) \cdot d E_{\text {Ion }} / \int \dot{N}_{\text {Ion }}\left(E_{\text {Ion }}\right) \cdot d E_{\text {Ion }} \\
& \overline{\left\langle\sigma_{P h}\right\rangle_{e}} \equiv\left(N_{P h} / N_{e}\right) \cdot \overline{\left\langle 1+\sigma_{P h}\right\rangle} \equiv\left(N_{P h} / N_{e}\right) \cdot \int\left[1+\sigma_{P h}\left(E_{P h}\right)\right] \cdot \dot{N}_{P h}\left(E_{P h}\right) \cdot d E_{P h} / \int \dot{N}_{P h}\left(E_{P h}\right) \cdot d E_{P h}
\end{aligned}
$$

Similar definitions hold for $N_{I o n} \equiv \int N_{I o n}\left(E_{I o n}\right) \cdot d E_{I o n}$ and $N_{P h} \equiv \int N_{P h}\left(E_{P h}\right) \cdot d E_{P h}$, as well as the average ion-induced and photon-induced yields weighted by their respective incident flux distributions. The formulation of Eqs. (3) to (5) is similar to that developed by Lai and Della-Rose, where their averages are weighted by particle energy. ${ }^{24}$

Consider the common limiting case in which the terms of Eqs. (3) and (4) in curly brackets are negligible, for example when $N_{P h} \rightarrow 0$ in eclipse and $N_{I o n} \ll N_{e}$. Then the magnitude of the overall spacecraft charge is determined (assuming an energy independent flux, $N_{e}$ ) by the area under the $\sigma_{e}$ yield curve and above 1 found between the crossover energies as compared to the area between 1 and $\sigma_{e}$ found below $E_{l}$ and above $E_{2}$ (see shaded areas in Fig. (2)). The crossover energies, $E_{l}$ and $E_{2}$, are energies at which the total electron yield, $\sigma_{e} \equiv \delta+\eta$, transitions from less than to greater than unity. When the ion- and photo-yields are non-negligible. Lai noted that the horizontal line, $\sigma_{e} \equiv \delta+\eta=1$, shifts downward as $N_{P h}$ increases. ${ }^{25}$ Similar shifts are possible due to increases in $N_{\text {Ion }}$, although these are usually much less pronounced, since the ion contribution is typically much less than the electron or photon contributions. When the horizontal line is shifted below zero, ion and photon contributions dominate and only positive charging can occur for any magnitude incident electron flux.

In the more general case with energy dependant differential fluxes, the crossover energies, $E_{1}$ and $E_{2}$, are energies at which the curves in Figs. 4(a) to 4(c) cross (e.g., when the weighted total electron yield, $\left[\delta\left(E_{e}\right)+\eta\left(E_{e}\right)\right] \cdot N_{e}\left(E_{e}\right)$, and the flux distribution, $N_{e}\left(E_{e}\right)$, are equal). Then, the shaded areas between the curves in Figs. 4(a) to 4(c) determine the sign and magnitude of the net charge. This is evident in the curves; as the electron flux distribution becomes more extreme, the area between the curves contributing to negative charging becomes larger.

Even modest changes in any one of the constituent functions in Eq. (3) can act to tip the balance from low-level positive charging to high-level negative charging. Positive [negative] charging occurs when the left-hand side is greater than [less than] the right-hand side; Eq. (3) is an equality at current equilibrium with an accumulated charge density of $Q_{n e t} / A$. If we express the constituent functions in Eq. (3) in terms of environmental and materials parameters, Eq. (3) can then be solved to determine the critical value of each parameter that satisfies the equality at equilibrium for threshold charging at zero potential (or net current). We now consider various scenarios that can affect these functions and trigger threshold charging.

\section{A. Changes of the Space Plasma Environment}

It is well known that temporal changes of the space plasma environment, as characterized in Eq. (3) by the flux distribution, $N_{e}\left(E_{e}\right)$, can cause abrupt changes in the spacecraft equilibrium potential. ${ }^{26-30}$ Two such mechanisms can result from convection of stable plasmas populations past the spacecraft, motion of the spacecraft with respect to magnetospheric convection boundaries, or injection of hot plasmas by substorms. First, we consider change in the shape of the energy distribution, $N_{e}\left(E_{e}\right)$, which increases the relative fraction of higher energy electrons above the crossover energy. Although any parametric model of the environment can lead to critical parameters at threshold, we limit our discussion here to a common parametric form of $N_{e}\left(E_{e}\right)$, the Maxwellian distribution

$$
\dot{N}_{e}\left(E_{e} ; n_{e}, T_{e}\right)=n_{e} \cdot\left(8 / \pi m_{e} k_{B} T_{e}\right)^{1 / 2}\left(E_{e} / k_{B} T_{e}\right) e^{-\left(E_{e} / k_{B} T_{e}\right)}
$$

where $n_{e}$ is the electron plasma density. An increase in the electron plasma temperature, $T_{e}$, modifies the shape of the $N_{e}\left(E_{e} ; T_{e}\right)$ distribution and can shift the Maxwellian distribution of the incident primary electrons to higher energies and diminish the number of incident low energy electrons between the crossover energies (see for example, the three distributions in Fig. 3). Increasing $T_{e}$ from a few thousand eV upward acts to decrease the flux-weighted integrals involving both $\delta$ and $\eta$ in Eq. (3), leading to a threshold charging effect. ${ }^{29,31}$ Indeed, Lai has identified a critical temperature for the electron flux at the onset of threshold charging as the root, $T_{e} \rightarrow T_{C r}$, of Eqs. (3) or (4) with $N_{e}\left(E_{e} ; T_{e}\right)$ expressed explicitly as a single or double Maxwellian with effective electron temperature(s) $T_{e}=T_{l}$ (and $\left.T_{2}\right){ }^{26,27}$ This has been confirmed by the detailed investigation of Olsen. ${ }^{30}$

Secondly, increases in the overall relative magnitude of the electron plasma density, $\mathrm{N}_{\mathrm{e}} / \mathrm{N}_{\text {Ion }}$ (or equivalently, $n_{e} / n_{\text {Ion }}$ )or $N_{e} / N_{P h}$, in Eq. (4) can cause a transition from positive to negative charging under certain favorable conditions, as was also observed by Olsen. ${ }^{30}$ Because both photoemission and ion-induced electron emission always 
produce positive charging, increasing $n_{e}$ can trigger threshold charging only if the initial total electron-induced yield by itself produces negative charging (i.e., $\overline{\langle\delta+\eta\rangle}<1$ ). By contrast, if $\overline{\langle\delta+\eta\rangle}>1$, no scaling of $\hat{N}_{e}\left(E_{e}\right)$ can lead to negative charging. ${ }^{11}$ In this case, increases in the electron plasma density typically lead to only minimal increases in positive surface bias, as a result of the self-regulating nature of positive biasing. ${ }^{19}$

Similar effects can occur if the magnitude or the shape of $N_{I o n}\left(E_{I o n}\right)$ or $N_{P h}\left(E_{P h}\right)$ are changed relative to $N_{e}\left(E_{e}\right)$. Changes in $\hat{N}_{\text {Ion }}\left(E_{\text {Ion }}\right)$ can result from changes in the plasma environment similar to those for $N_{e}\left(E_{e}\right)$, such as substorms, coronal mass ejections, and plasma boundaries. Significant changes in the relative magnitudes of the electron and ion fluxes can also results from the differences in transit times of particles from the solar activity. ${ }^{32}$ Modest changes in the solar irradiance, $N_{P h}\left(E_{P h}\right)$ can result from solar flares, solar storms, and solar cycles. ${ }^{32}$ Optical absorption of the atmosphere, which varies with altitude, density, composition and temperatures, can have dramatic effects on both magnitude and shape of $N_{P h}\left(E_{P h}\right) .{ }^{32}$ Modifications to $N_{P h}\left(E_{P h}\right)$ can also result from energy dependant filtering of reflected or transmitted sunlight from other surface adjacent to or above the absorbing surface, such as from solar concentrators, lenses and optics elements, coverglasses, anti-reflection coatings, or contamination films.

\section{B. Changes of the Environment/Spacecraft Interface}

The sign of the equilibrium potential can also be modified by temporal changes in the juxtaposition of the spacecraft to the environment, including spacecraft orbit, orientation, and geometry. For example, the distribution or magnitude of incident fluxes, $\mathrm{N}(\mathrm{E})$, can vary due to changes in spacecraft position as the spacecraft moves in or out of radiation belts or magnetospheres, or is eclipsed behind astronomical bodies. Alternately, the position of inhomogeneous flux distributions can change with time, for example in solar flares or magnetotail aberration. ${ }^{33}$ In general, this class of changes is complex and very specific to the particular mission; these effects are best investigated with detailed modeling codes such as NASCAP ${ }^{7,9,10}$ and SPENVIS. ${ }^{34}$

Electron yields can also be modified due to changes in the orientation of the spacecraft relative to incident radiation fluxes with a preferred direction, such as solar electromagnetic radiation, high energy proton or electron fluxes from the solar wind, or charged particles trapped in magnetic fields. The products in the integrals of Eq. (3) are then dot products between preferred flux direction and the surface normal. Material surfaces directly facing the sun have full emission; surfaces facing away from the preferred direction, which are shaded in eclipse, have their incident fluxes "turned off". Between these extremes, the fluxes, N(E), incident on a smooth flat surface decrease with the exposed cross sectional area as $\cos (\varphi)$, where $\varphi$ is the angle of incidence of the flux from the surface normal. However, non-normal incidence electron yields of both photoemission at constant reflectivity ${ }^{25,35}$ and secondary electron emission ${ }^{36}$ increase approximately as $1 / \cos (\varphi)$. To first order, this results from the fact that the higher energy incident electrons and photons have significantly longer mean free paths than the low energy secondary electrons, which results in most emitted SE being produced near the surface (typically within 5-10 nm) in a small fraction of the penetration region (typically $10^{2}$ to $10^{4} \mathrm{~nm}$ deep). At more grazing angles the incident particles spend a larger fraction of their trajectory near the surface, where the isotropic SE produced can subsequently escape. This argument breaks down at large $\varphi$, where the penetration depth of the incident photons and electrons is comparable to or less than the mean free path of the SE and emission plateaus. This argument also fails for all but very high energy ions, since ion mean free paths are much less than the mean free paths of electrons of comparable energies. High energy BSE are also exceptions to this $1 / \cos (\varphi)$ enhancement, since incident and emitted electrons have comparable mean free paths; in general, quasieleastic BSE emission and inelastic BSE emission are comprised to varying degrees by one component following a nearly $1 / \cos (\varphi)$ dependence and another that is more tightly focused about the specularly reflected emission beam (in analogy to optical diffuse and specular reflection) ${ }^{36}$ (Note that the dependence of photon absorptivity on incidence angle can also affect charging, ${ }^{25}$ these effects are discussed below in relation to Eq. (7).)

Satellite geometries unique to specific missions and spacecraft can also have effects. For example, shadowing of one part of the spacecraft by another part can mimic eclipse. Differential charging of one part of the satellite relative to adjacent parts can act to modify the redistribution of emitted low energy charged particles traversing the plasma environment and their subsequent (re)capture by surfaces. Differential charging between surfaces can lead to the formation of potential wells that trap low energy emitted electrons and potential barriers that block reabsorption of the low energy emitted electrons [Ref. 25 and references therein]. Lai et $a l^{25,37,38}$ and others ${ }^{39-41}$ have investigated in detail a simple monopole-dipole form of differential charging and shown that in such cases the critical temperature for threshold charging is approximately the same as in eclipse. Lai et al has extended this to a monopole-quadrupole model to include the effects of a satellite spinning faster than the equilibration time of the satellite potential and reached a similar conclusion about $\mathrm{T}_{\mathrm{Cr} .}{ }^{25,35}$ 
Table 1. Electron Emission Fitting Parameters and Equilibrium Potentials in an "Extreme" Environment"

\begin{tabular}{|c|c|c|c|c|c|c|c|c|c|c|c|}
\hline \multirow{2}{*}{ Material } & \multicolumn{5}{|c|}{ SE Emission } & \multirow{2}{*}{$\frac{B S E}{Z_{e f f}}$} & \multicolumn{2}{|c|}{ Ion Emission } & \multirow{2}{*}{$\begin{array}{c}\text { Photoyield } \\
\sigma_{\text {solar }} \\
\left(\mu \mathrm{A}-\mathrm{m}^{-2}\right)\end{array}$} & \multirow{2}{*}{$\begin{array}{c}\text { Eq Pot. } \\
(\mathrm{eV})\end{array}$} & \multirow{2}{*}{$\frac{\text { Figure. }}{\text { Ref. }}$} \\
\hline & $\begin{array}{c}\delta_{\max } \\
(\mathrm{el} / \mathrm{el})\end{array}$ & $\begin{array}{l}E_{\max } \\
(\mathrm{eV})\end{array}$ & $n_{1}$ & $n_{2}$ & $b_{1} / b_{2}$ & & $\begin{array}{c}\sigma_{\max }^{H} \\
\text { (el/ion) }\end{array}$ & $\begin{array}{l}E^{H}{ }_{\text {max }} \\
(\mathrm{keV})\end{array}$ & & & \\
\hline $\mathrm{Au}(5$ param $)$ & 1.49 & 600 & 1.85 & 0.65 & 3.6 & 50.9 & 0.336 & 1238 & 36.4 & +0.34 & $2(\mathrm{a}),[8]$ \\
\hline $\mathrm{Au}$ (variable $\mathrm{n}$ ) & 1.47 & 569 & & NA & NA & 50.9 & 0.336 & 1238 & 36.4 & +1.1 & $2(\mathrm{a}),[8]$ \\
\hline $\mathrm{Au}$ (Young) & 1.44 & 525 & & NA & NA & 50.9 & 0.336 & 1238 & 36.4 & +1.3 & $2(\mathrm{a}),[8]$ \\
\hline $\mathrm{Au}$ (Feldmann) & 1.61 & 686 & NA & NA & NA & 50.9 & 0.336 & 1238 & 36.4 & -25800 & $2(\mathrm{a}),[8]$ \\
\hline $\mathrm{Au}$ (Sternglass) & 1.52 & 700 & NA & NA & NA & 50.9 & 0.336 & 1238 & 36.4 & NA & $2(\mathrm{a}),[8]$ \\
\hline PI (Kapton H) & 2.22 & 350 & 2.1 & 0.71 & 1.8 & 10.4 & 0.37 & 1238 & 36.4 & NA & 1(a),[8] \\
\hline PI $\left(\sim 4 \mathrm{pC} / \mathrm{mm}^{2}\right)$ & 2.19 & 250 & 2.3 & 0.9 & 2.3 & 10.4 & 0.37 & 1238 & 36.4 & -8734 & $3(d)$ \\
\hline PI $\left(\sim 1 \mathrm{pC} / \mathrm{mm}^{2}\right)$ & 1.74 & 250 & 2.6 & 0.9 & 6.0 & 10.4 & 0.37 & 1238 & 36.4 & -9308 & $3(d)$ \\
\hline $\mathrm{PI}\left(\sim 0.4 \mathrm{pC} / \mathrm{mm}^{2}\right)$ & 1.34 & 300 & 2.4 & 0.9 & 2.5 & 10.4 & 0.37 & 1238 & 36.4 & -9432 & $3(d)$ \\
\hline PI $\left(\sim 3 \mathrm{fC} / \mathrm{mm}^{2}\right)$ & 1.08 & 250 & 1.3 & 0.9 & 2.5 & 10.4 & 0.37 & 1238 & 36.4 & +1 & $3(d)$ \\
\hline Graphite (HOPG) & 1.21 & 240 & 1.29 & NA & NA & 6.0 & 0.50 & 261 & 1.78 & -6287 & [8] \\
\hline g-C $\left(1050^{\circ} \mathrm{C}\right)$ & 1.22 & 300 & 2.0 & 0.8 & 1.8 & 4.3 & 0.36 & 500 & 7.9 & -30100 & [58] \\
\hline $\mathrm{g}-\mathrm{C}\left(850^{\circ} \mathrm{C}\right)$ & 1.41 & 200 & 1.8 & 0.8 & 1.8 & 5.5 & 0.37 & 450 & 7.9 & NA & {$[58]$} \\
\hline $\mathrm{g}-\mathrm{C}\left(650^{\circ} \mathrm{C}\right)$ & 1.24 & 200 & 2.0 & 0.8 & 18 & 5.3 & 0.38 & 424 & 7.9 & -29300 & [58] \\
\hline $\mathrm{g}-\mathrm{C}\left(350^{\circ} \mathrm{C}\right)$ & 1.34 & 250 & 1.8 & 0.8 & 1.8 & 5.8 & 0.29 & 817 & 7.9 & -27200 & [58] \\
\hline g-C (unannealed) & 1.74 & 240 & 1.61 & NA & NA & 13.8 & 0.57 & 30 & 7.9 & -29300 & [58] \\
\hline
\end{tabular}

* Evaluated for an "Extreme" ATS-6 environment as defined in Fig. 4.

Similarly, differential charging can be modified by the internal redistribution of accumulated charge as controlled by the resistivity of the spacecraft materials. ${ }^{42}$ Dennison et al found in their study of differential charging of contaminated surfaces that there was a critical resistivity necessary to trigger differential threshold charging of more complex geometries involving multiple conducting and insulating surfaces. ${ }^{43}$ Lai also discussed the effects of resistivity on threshold charging. ${ }^{25}$ Resistivity itself can evolve with time, for example due to contamination and oxidation, accumulation of charge, changes in incident radiation dose rates, or radiation damage of materials.

\section{Changes in Electron Emission of Spacecraft Materials}

Finally, the primary focus of this paper is how changes in the spacecraft materials electron emission properties can also lead to a threshold effect for spacecraft charging. ${ }^{11,43}$ These effects enter into Eq. (3) through the electron yields $\delta, \eta, \sigma_{I o n}$, and $\sigma_{P h}$. For changes in both $\delta$ and $\eta$ yield curves, the possible threshold scenarios are much richer, since electron-induced emission can cause both positive and negative charging.

Most obviously, a $\delta$ or $\eta$ yield curve shape and magnitude can be modified if the spacecraft surface is changed from one material to another. This drastic approach is often used to mitigate spacecraft charging issues. Extensive databases of electron emission curves have been compiled, ${ }^{8,44}$ although most materials (especially insulators) remain poorly characterized or unstudied and very little information exists as to how materials behave at non-ambient conditions. Because electron emission is a surface phenomenon, ongoing surface modification can be viewed as a gradual change from one material to another. Similarly, layered materials with thicknesses less than the mean free path of the incident particles can exhibit electron yield curves that are combinations of the yield curves of the constituent materials. ${ }^{8}$

Inaccuracies and systematic errors in measurements of the yield curves can lead to errors in the determination of equilibrium charging. Absolute yield is difficult to measure, particularly for insulators. ${ }^{18,20,21}$ Error in absolute yield calibration magnifies the yield curve in the vertical direction; it is equivalent to an error in $\delta_{\max }$. Error in the incident particle energy due to miscalibration of the instrumentation or offset potentials in the source, sample or detector act to shift the yield curve in the horizontal direction. Such miscalibrations are similar in effect to potential shifts due to biasing of conductor samples or charge accumulation in insulators (see below). Energy miscalibration introduces equivalent offset errors in $E_{\max }$ and the crossover energies.

Errors can also be introduced into the yields from the imperfect parameterization of data for use in charging codes. To incorporate electron yield curves into spacecraft charging codes such as NASCAP- $2 \mathrm{k}^{7,9,10}$ and SPENVIS ${ }^{34}$ requires that measured SE yield curves be fit to parametric equations. Different SE yield models vary most in the way the incident electron penetration and energy dissipation inside the material is modeled, and also in the way they account for the propagation of secondary electrons. Selecting the most appropriate model determines the shape of the yield curve and is critical to accurately represent the materials properties in the charging codes. These models are 
successful to varying degrees for conductors and insulators in fitting the rising edge below $\delta_{\max }$, the peak at $\delta_{\max }$, and the crossover energies (see Fig. 2(a)). They show their largest differences in their asymptotic dependence at high energy, which is of particular importance in determining the negative charging resulting from this region of low SE yield. ${ }^{45}$

Increased surface roughness affects electron emission in various ways. For very shallow relief, roughening can increase emission by increasing the emitting area and by causing more grazing incidence. By contrast, deeper roughening can reduce $\mathrm{SE}$ emission and inhibit negative charging by reabsorbing emitted electrons, when the roughness is on the order of the SE mean free path $\left(\sim 10^{0} \mathrm{~nm}\right)$ or larger. ${ }^{17,36}$ Such roughening has been suggested as a mitigation strategy to limit "snapover" discharge believed to be at least partially caused by SE emission of insulating surfaces. ${ }^{46}$ As an extreme example, a material comprised of very deep features with very thin walls acts essentially as a Faraday cup, effectively trapping all SE and BSE electrons so that $\sigma_{E l} \rightarrow 0$ and only positive charging can occur.

Sample potential also affects electron emission in complex ways. The example of charge accumulation provides an interesting counterexample to the conjecture that the environmental and materials aspects of the problem in Eq. (3) have been separated into the fluxes and yield functions, respectively. Accumulated charge directly affects the incident flux, the fraction of the emitted charge that is reabsorbed, the electron yield via changes in $\mathrm{PE}$ penetration and SE production, transport and emission, and even the charge redistribution rate through the dark current ${ }^{42}$ and radiation-induced conductivities. ${ }^{47}$ Biasing of conducting samples effectively acts to shift the emission spectra in the horizontal direction, as electrons emitted from a negatively biased surface are given a boost in kinetic energies and electrons emitted from a positively biased surface are retarded (see Fig. 2(b)). ${ }^{19}$ In addition, traversing from a lower [higher] potential region to a higher [lower] potential region acts to deflect electron trajectories toward [away from] the surface normal, in a manner reminiscent of optical refraction. ${ }^{36}$ Thus, sample bias can modify the currents returning to the emitting or adjacent surfaces. ${ }^{19}$ Because charge resides at the surface of a conductor, there are no internal electric fields and it is only the electrons that cross the surface barrier and are emitted that are affected. There are no changes in the production and transport within the conductor. By contrast, charge within an insulator sets up internal electric fields which can modify the PE and SE transport and the PE energy loss or SE production mechanisms.

Electron emission can also be changed by effects that modify the electronic band structure of the material, and hence the production of SE and the transport of PE and SE within the material. Grais ${ }^{57}$ discussed the effect of bandgap on yields of large bandgap semiconductors and Corbridge ${ }^{58}$ has studied the effects for low energy bandgaps. One type of these changes result from modification of the occupation of electron states; typically valence or trapped states are excited into conducting states. For example, increased temperature increases the population of electron-hole pairs and can populate or depopulate localized states. Concurrent fluxes of higher energy photon or particle radiation can cause similar changes in the distribution of occupied electron energy states within the material. This directly affects conductivity and redistribution of spacecraft charging. It also affects the probability of PE or SE energy loss mechanisms that determine electron mean free path. In the extreme, large band gap semiconductors have large yields since low energy SE produced deep with the material can escape as the lack of low energy loss mechanisms make the insulator effectively transparent to low energy electrons with energies up to the bandgap energy.

Another type of bandgap modification results from the change of the actual bandstructure of the material. Changes in the atomic scale structure of a material, due for example to thermal annealing or phase transitions, can 
directly modify the bandstructure. Doping, contamination or surface modification can produce localized states within the bandgap. Larger fluences of high energy radiation can produce additional trap sites within the material and cause other radiation damage effects.

Modification of $\sigma_{I o n}$ or $\sigma_{P h}$ can alter the relative amount of positive charging due to ions or photons, respectively, and induce threshold charging in certain favorable cases, in a manner similar to changes in the electron plasma density. As noted in Section II.A, an increase in either $\overline{\left\langle 1+\sigma_{I o n}\right\rangle_{e}}, \overline{\left\langle\sigma_{P h}\right\rangle_{e}}$ in Eq. (4) [which can be due equivalently to increases in either $\overline{\left\langle\sigma_{I o n}\right\rangle}$ or $\overline{\left\langle\sigma_{P h}\right\rangle}$ or in $N_{I o n} / N_{e}$ or $N_{P h} / N_{e}$ ] reduces the yield weighted-flux curves, such as those in Figs. 4(a), (c) and (e), thereby shifting the crossover energies to wider spaced values (analogous to reducing the horizontal line that determines the effective crossover energies in $\langle\delta+\eta\rangle$ curves, such as those in Fig. 1), and effectively increasing $\mathrm{T}_{\mathrm{Cr}}$. Changes in $\sigma_{\text {Ion }}$ can trigger such threshold charging, although these are usually much less pronounced, since the ion yield is typically much less than $\sigma_{e}$ or $\sigma_{P h}$. Changes in $\sigma_{I o n}$, like changes in $\delta$, are highly surface sensitive and can result from oxidation or even monolayer contamination, surface roughness, angle of incidence or charge accumulation.

More interesting are changes in $\sigma_{P h}$. Based on arguments outlined by Lai ${ }^{25}$

$$
\overline{\left\langle\sigma_{P h}\right\rangle_{e}}=\frac{1}{N_{e}^{T o t}} \int\left[\dot{N}_{P h}\left(E_{P h}\right) \cos (\varphi)\right]\left[\frac{\sigma_{P h}^{n}\left(E_{P h}\right)}{\cos (\varphi)}\right]\left\{\left\{1-\mathrm{R}_{h}\left(E_{P h}\right)-\mathrm{T}_{n}\left(E_{P h}\right)\right] \cos (\varphi)\right\} d E_{P h}
$$

The first term in the integral is related to the decrease in cross sectional area with angle of incidence $\varphi$, discussed above. The second term is the enhancement of the photoyield for normal incidence, $\sigma_{P h}{ }^{n}\left(E_{P h}\right)$, due to photon penetration depth. The last term in curly brackets is the optical absorptivity, $A_{n}$, that corrects $\overline{\left\langle\sigma_{P h}\right\rangle_{e}}$ for the fact that only absorbed photons deposit energy in the material and can thereby produce photoelectrons. To a reasonable approximation, the absorptivity is equal to one minus the sum of the reflectivity plus transmission at normal incidence all scaled by the $\cos (\varphi){ }^{25,35}$ The inherent absorptivity and transmissivity at normal incidence are complex properties of the microscopic bandstructure and macroscopic dielectric properties of the material and depends heavily on the incident photon energy. These can be changed by structural changes in the material, or through UV or radiation damage. Reflectivity at normal incidence depends more critically on surface modifications (on the order of incident optical wavelengths), through changes in surface roughness (see above), contamination, surface degradation (e.g., from atomic oxygen), or temperature.

\section{Quantifying Threshold Charging}

An important question then is how to quantitatively gauge the relative effects of the various changes identified in Sec. II on threshold charging. Equation (3) provides an analytic expression to determine the equilibrium potential, if the space environment, spacecraft/environment interface, and materials properties are all fully characterized. The most straightforward approach is to determine the equilibrium potential of panels of representative spacecraft materials under a range of environmental conditions as a function of change in these parameterized causes, so as to determine the fractional change in the parameters necessary to induce threshold charging. ${ }^{11}$ Examples of this approach include:

(i) plots of spacecraft potential versus of optical absorptivity (Fig. 5), SE yield amplitude (Fig. 6), and contamination (Fig. 7 of Ref. 11), and similar plots of space environment characteristics $\left(T_{e}, n_{e}, T_{\text {Ion }}, n_{\text {Ion }}\right.$ in Ref. 24 and $T_{e}$ in Ref 25) and resistivity (Ref. 43);

(ii) mapping out the simultaneous combinations of two parameters in Eq. (3) that lead to threshold charging and zero equilibrium potential. Examples of this approach include contamination versus space environment characteristics and SE and BSE yield parameters (Eq. 9 of Ref. 11) and reflectivity versus electron temperature (Ref 25);

(iii) a less direct approach to consider how one materials property varies with change in another parameterized material property already know to lead to threshold charging and to then infer how threshold charging depends on the initial property. This is done for crossover energies vs $\delta_{\max }($ Fig. 7) and bandgap vs $\delta_{\max }$ (Fig. 8).

We have modeled the absolute charging behavior of a hypothetical flat, two-dimensional satellite panel of a single material as it undergoes modification and the concomitant changes in spacecraft charging. Calculations are presented here for the conductor $\mathrm{Au}$ (polycrystalline, $4 \mathrm{~N}$ purity, $25 \mu \mathrm{m}$ thick, $\sim 8 \mathrm{~nm}$ rms surface roughness, ESPI) and the polymeric polyimide Kapton ${ }^{\mathrm{TM}} \mathrm{H}(13 \mu \mathrm{m}$ thick on $100 \mathrm{~nm}$ thick vapor deposited Al, Sheldahl, type 
$146446 / \mathrm{G} 405110$ ), based on various fits to measured yield curve data. Charging modeling was done using the NASCAP Interactive Spacecraft Charging Handbook. ${ }^{10}$ Standard NASCAP values, as listed in the SEE Charge Collector Database, ${ }^{8}$ were used for all materials parameters except those six related to $\mathrm{SE}$ and BSE emission. The specific values used for various simulations are listed in Table I. Three representative geosynchronous orbit environments incorporated into NASCAP were considered as described in detail in Fig. 4. Full sunlight and full shade (eclipse) were also considered, in effect turning on and off the photo-induced electron emission.

The causes investigated include:

\section{A. Changes in the electron environment.}

Quantitative studies of the effects of environmental parameters of threshold charging in Refs. 11,25 and 26 have consistently confirmed the observations made by Olsen. ${ }^{30}$ and others. Figure 9 of Chang ${ }^{11}$ quantitatively demonstrates the relative sensitivity to threshold charging for plasma temperature and plasma density. Negative charging becomes less extreme as the effective temperature of the electron distribution is reduced. As the electron flux (relative to ion or photon flux) is reduced, the negative charging becomes less severe. Below a nominal level, no amount of electron flux is sufficient to cause negative charging.

\section{B. Surface contamination.}

Studies by Davies ${ }^{48,49}$ and Chang ${ }^{11}$ showed that modest surface contamination can lead to significant changes in the SE and BSE emission of spacecraft surfaces. They found that surface oxidation and accumulation of monolayers of disordered carbon contamination decreased the yield curve amplitudes of $\mathrm{Au}$ and $\mathrm{Al}$ surfaces by about a factor of 2, and that these changes lead to threshold charging in eclipse. They argued that the formation of monolayers of disordered graphitic carbon was similar to accumulation of organic films on spacecraft surfaces, with subsequent cracking of the organics by UV or particle radiation. Figures 7 and 9 of Chang $^{11}$ illustrate the critical contamination level necessary to trigger threshold charging; in the specific case studied the critical value was only about two monolayers of carbon. The reverse situation has also occurred on a probe of the Plasma Diagnositics Package, which flew on shuttle flight STS-3; atomic oxygen stripped a colloidal graphite (Aquadag ${ }^{\mathrm{TM}}$ ) coating from an $\sim 8 \mathrm{~cm}$ diameter spherical Au surface. ${ }^{55}$ Dennison studied differential charging due to organic film deposition on satellite surfaces and the effects of resistivity. ${ }^{43}$

\section{Selection of electron yield model.}

Under suitable circumstances, threshold charging depends on the yield model used. This is illustrated for the Au SE yield curve in Fig. 1(b), with fitting parameters listed in Table I. The models considered include a Bethe theory model by Sternglass ${ }^{50,51}$ and three empirical single power-law models [Young $^{52}$, with $n=1.35$; Feldman, ${ }^{53}$ and a variable $n$ model $^{54}$. We also included the standard empirical double power-law model SE fit used in NASCAP, ${ }^{7}$ with five fitting parameters $\delta_{\max }, E_{\max } n_{1}, n_{2}$, and $b_{1} / b_{2}$. Figure 1(a) shows that these models predict significant differences in the yield at energies above $\delta_{\max }$, and in particular at energies above $E_{2}$ that contribute to negative charging. Table I lists the equilibrium potentials of a Au panel, calculated in the "extreme" environment in eclipse (see Fig. 4). There are modest differences evident in the small positive potentials for the fitting parameters of the power law fits and a very large change to a large negative potential for the Feldman fit.

Chang $^{11}$ quantitatively investigated the relative effects of variations in the standard NASCAP fitting parameters $\delta_{\max }, E_{\max }$ and $n$ for a single, variable-exponent power law model of the SE yield curve and $Z_{\text {eff }}$ for a single parameter model of the BSE yield curve. The study found that:

(i) as either $\delta_{\max }$ or $E_{\max }$ decreased, the negative charging became more extreme; 
(ii) increases in the power law exponent reduced the yield at higher energies, thereby enhancing negative charging; and

(iii) reduction in $Z_{\text {eff }}$ (or equivalently total $\mathrm{BSE}$ yield) resulted in enhanced negative charging.

Figure 9 of Chang $^{11}$ quantitatively demonstrated the relative sensitivity to threshold charging of these parameters.

\section{Absolute yield.}

The effects of change in maximum SE yield, $\delta_{\max }$, on equilibrium potential are shown in Fig. 6 for the representative three test environments. As expected, reduction of $\delta_{\max }$, with all other parameters fixed, led to threshold charging. For a $\mathrm{Au}$ panel in eclipse, $\delta_{\max }$ at threshold is approximately $87 \%, 67 \%$ and $34 \%$ of the measured maximum yield of 1.49 , for the environments respectively. This is consistent with similar studies under different conditions described in Refs. 11, 43, and 48.

\section{E. Crossover Energies.}

Since we have shown that changes in $\delta_{\max }$ affect threshold charging, it follows that change in the crossover energies will also affect threshold charging. Figure 7 shows the relative variations of $E_{1}$ and $E_{2}$ as $\delta_{\max }$ is modified for Au.

\section{F. Insulator Charging.}

There is ample evidence that charge accumulation in insulators affects yields (See for example, Figure 3(a) and the discussion in Sec. II.C.). Indeed, evidence from low fluence, pulsed measurements of insulator yields suggests that very small incident fluences, as small as $\sim 3 \mathrm{fC} / \mathrm{mm}^{2}$ or $\sim 2 \cdot 10^{4}$ electrons $/ \mathrm{mm}^{2}$, can have large effects on the yields. ${ }^{18,20,21}$ We found at higher fluences that the yield curves of insulators flattened as the sample was charged more by the charge in the incident pulse, with the yield at the crossover energies pinned to unity where not charging occured. This is illustrated by two yield curves shown in Fig. 3(a), taken at fluences per pulse of $\sim 0.4 \mathrm{pC} / \mathrm{pulse}(\sim 80$ $\left.\mathrm{pC} / \mathrm{mm}^{2}\right)$ and $\sim 3 \mathrm{pC} /$ pulse $\left(\sim 600 \mathrm{pC} / \mathrm{mm}^{2}\right)$, respectively. To quantify this effect, we have measured a series of yield decay curves for Kapton, as shown in Fig. 3(c). These curves measure the total yield at fixed incident energy between the crossover energies $(100 \mathrm{eV}, 250 \mathrm{eV}, 300 \mathrm{eV}, 400 \mathrm{eV}$ and $500 \mathrm{eV})$ with repeated pulses of very low fluence. For very highly insulating materials such as Kapton ${ }^{56}$ very little incident charge is dissipated during the duration of the measurements. As charge accumulates, total yield tends to unity. The evolution of these yield curves was then used to determine the yields from these decay curve energies at a series of charge fluence densities $\left(\sim 4 \mathrm{pfC} / \mathrm{mm}^{2}, \sim 1 \mathrm{pC} / \mathrm{mm}^{2}, \sim 0.4 \mathrm{pC} / \mathrm{mm}^{2}\right.$, and $\left.\sim 3 \mathrm{fC} / \mathrm{mm}^{2}\right)$; Fig. 3(d) shows the estimated total yield curves based on these values at fluence densities. Finally, the estimated total yield curves at various fluence densities (minus a fixed BSE yield component) were fit with five parameter NASCAP SE yield functions (see Table I) and equilibrium potentials for panels of Kapton in the "extreme" environment in eclipse were calculated. A critical value of charging for threshold charging was observed; at a fluence density of $\sim 3 \mathrm{fC} / \mathrm{mm}^{2}$ and below the simulation predicted small positive charging, while at $\sim 400 \mathrm{fC} / \mathrm{mm}^{2}$ amd above many kilovolts negative charging was predicted.

\section{G. Bandstructure.}

A study by Corbridge looked at the effects of modification of the bandstructure of graphitic carbon on the electron yield. ${ }^{58}$ Graphitic carbon has one of the lowest know total electron yields and is often used as a surface coating when low yield surfaces are required in applications such as electron microscopy of plasma confinement. Crystalline graphite is a zero bandgap semimetal; the bandgap increases as disorder is introduced into the atomic structure. ${ }^{59}$ (At an extreme limit, diamond - with a bandgap of $5.4 \mathrm{eV}$ - is known to have extremely high electron yields.) Starting with an amorphous form of graphitic carbon, $\mathrm{g}-\mathrm{C}$, thermal annealing can gradually increase the atomic order towards that of crystalline (HOPG) graphite. This annealing process is found to decrease the g-C 
bandgap from $\sim 0.6 \mathrm{eV}$ to $\sim 0.1 \mathrm{eV}$ through successively higher annealing temperatures, while maintaining many other materials properties that are known to affect electron emission such as average atomic number, surface roughness, and density. Figure 8 shows the measured $\delta_{\max }$ as a function of carbon bandgap energy; complete fitting parameters for the six carbon samples are listed in Table I. Maximum SE yield clearly increases with increasing bandgap energy; surprisingly this increase is seen to be nearly linear, at least for this experiment on graphitic carbon. Table I lists the equilibrium potentials of a panel of each of the graphitic carbon materials, calculated in the "extreme" environment in eclipse (see Fig. 4). A critical value of bandap energy (or annealing temperature) for threshold charging is observed; below a bandgap energy of $\sim 0.25$

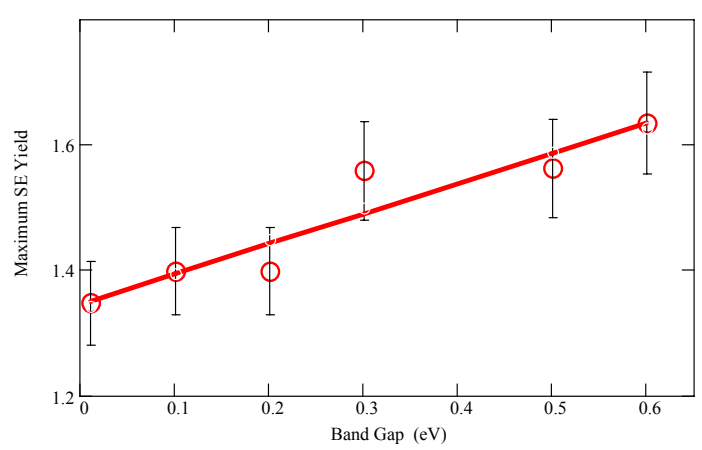

Figure 8. Maximum secondary electron yield, $\delta_{\max }$, as a function of bandgap energy for thermally annealed graphitic amorphous carbon.

$\mathrm{eV}$ (above an annealing temperature of $\sim 500{ }^{\circ} \mathrm{C}$ )

negative charging is predicted, while above $\sim 0.25 \mathrm{eV}$ (below $\sim 500^{\circ} \mathrm{C}$ ) positive charging is predicted.

\section{H. Absorptivity.}

Under suitable circumstances, an increase in optical absorptivity can lead to threshold charging at finite values. Figure 5 shows the calculated equilibrium potential of a flat panel of $\mathrm{Au}$ for three specific conditions. In full sunlight these panels exhibit positive charging. However, as the absorbtivity is reduce below from $2 \%$ to $0.2 \%$ (depending on specific conditions), the panel undergoes threshold charging. These results confirm the predictions of Lai, who calculated the critical temperature as a function of the reflectivity for several materials. ${ }^{25}$

\section{Conclusion}

In this paper we have explored how modest changes in spacecraft charging conditions can lead to threshold charging. These changes were discussed in terms of a general framework for threshold charging, based on simple consideration of charge conservation at spacecraft equilibrium in simple scenarios. Changes in the space plasma environment that lead to threshold charging were reviewed. Threshold charging resulting from changes in the juxtaposition of the spacecraft to the environment, including spacecraft orbit, orientation, and geometry were explored. We focused on the effects of possible changes in electron emission properties of representative spacecraft materials. These included changes of contaminated and layered materials; biasing and charge accumulation; bandstructure occupation and density of states caused by heat, optical or particle radiation; optical reflectivity and absorptivity; and inaccuracies and errors in measurements and parameterization of materials properties. We quantitatively gauged the relative extent to which these various changes in electron emission alter a spacecraft's charging behavior and lead to threshold charging for a number of specific cases.

The implication of these results for real spacecraft is obvious and of critical importance. In many cases, it may not be sufficient to model spacecraft charging of only pristine surfaces during the design phase of spacecraft development. Modest modifications of a variety of conditions can lead to significant changes in the electron emission of spacecraft surfaces. In geosynchronous environments, eclipsed surfaces can see dramatic changes in absolute charging and develop kilovolt negative biases. Of particular concern is the possibility of large differential charging between modified and unmodified surfaces that develop as the electron emission of surfaces evolve. To investigate the effects of modifications of materials properties on charging, it is essential to be able to accurately model the materials properties in charging codes such as NASCAP and SPENVIS. Of particular importance is the need to have better parametric models of the electron emission properties and to include the ability to model an incident photon spectrum other than the A0 solar spectrum and the full photoyield energy spectrum of materials. Increased capabilities to perform charging calculations in a batch mode over a range of parameters and to deal with layered materials of conductors and insulators would also be of great use in such studies of changing materials properties.

\section{Acknowledgments}

The authors would like to thank Henry Garrett and Eric Held for useful discussions. Measurements of disordered carbon were made by Jodie Corbridge, with additional help from Clint Thomson. Much of the support for this work 
was from the NASA Space Environments and Effects (SEE) Program, with additional support from the NASA Rocky Mountain Space Grant Consortium and the USU Vice President for Research Office.

\section{References}

\footnotetext{
${ }^{1}$ Hastings, D. and Garrett, H., Spacecraft-Environment Interactions, Cambridge University Press, Cambridge, 1996.
}

${ }^{2}$ Leach, R.D. and M.B. Alexander, "Failures and Anomalies Attributed to Spacecraft Charging," NASA Ref. Pub. 1375, Marshall Space Flight Center, 1995

${ }^{3}$ Garrett, H.B., The charging of spacecraft surfaces, Rev. of Geophys. and Space Phys., Vol. 19, No. 4, 1981, pp. 577-616.

${ }^{4}$ Garrett, H.B., Air Force Geophysical Laboratory, Rept. AFGL-TR-78-0116, Hanscom Air Force Base, MA, 1978.

${ }^{5}$ Katz, I.M, D.E. Parks, M.J. Mandell, J.M. Harvey, D.H. Brownwell, Jr., S.S. Wang and M. Rotenberg, "A Three Dimensional Study of Electrostatic Charging in Materials," NASA, NASA CR-135256, Greenbelt, MD, 1977.

${ }^{6}$ Katz, I., M. Mandell, G. Jongeward, and M.S. Gussenhoven, "The importance of accurate secondary electron yields in modeling spacecraft charging," J. Geophys. Res., Vol. 91, No. A12, 1986, pp. 13,739-13,744.

${ }_{7}^{7}$ Mandell, M.J., P.R. Stannard and I. Katz, “NASCAP Programmer's Reference Manual,” NASA Lewis Research Center, May 1993.

${ }^{8}$ Dennison, JR, Abbott, Jonathan, Hoffmann, Ryan, Sim, Alec, Thomson, C.D., and Corbridge, Jodie, "Electronic Properties of Materials with Application to Spacecraft Charging," Charge Collector Database [online database], NASA Space Environments and Effects Program, NASA Marshal Space Flight Center, October, 2005. URL: http://see.msfc.nasa.gov/ee/db_chargecollector.htm. [cited January 10, 2007].

${ }^{9}$ Mandell, M. J., Davis, V.A., Cooke, D. L., and Wheelock, A.T., "Nascap-2k Spacecraft Charging Code Overview," Proceedings of the $9^{\text {th }}$ Spacecraft Charging Technology Conference, EPOCHAL TSUKUBA, TSUKUBA, April 4-8, 2005.

${ }^{10}$ NASCAP Interactive Spacecraft Charging Handbook, Software Package, Beta Version 2.0, Maxwell Technologies, San Diego , CA, September, 1999.)

${ }^{11}$ Chang, W.Y., Dennison, J.R., Kite, Jason and Davies, R.E., "Effects of Evolving Surface Contamination on Spacecraft Charging," Proceedings of the $38^{\text {th }}$ AIAA Meeting on Aerospace Sciences, Reno, NV, 2000.

${ }^{12}$ Abbott, Jonathan, Hoffman, Ryan, and Dennison, JR, "Determination of Electron Emission Crossover Energies in Insulators," American Physical Society March Meeting 2006, Baltimore, MD, Bull. Am. Phys. Soc. Vol. 51, No. 1 Part II, March 13-17, 2006.

${ }^{13}$ Benka, O., A. Schinner, T. Fink, and M. Pfaffenlehner, "Electron-Emission Yield of Al, Cu, and Au for the Impact of Swift Bare Light Ions", Phys. Rev. A, Vol. 52, No. 5, 1995, pp. 3959-3965.

${ }^{14}$ Eder, H., M. Vana, F. Aumayr, and H. P. Winter, "Precise Total Electron Yield Measurements for Impact of Singly or Multiply Charged Ions on Clean Solid Surfaces", Rev. Sci. Instrum., Vol. 68. No. 1, 1997, pp. 165-169.

${ }^{15}$ Feuerbacher, B. and Fitton, B., "Experimental Investigation of Photoemission from Satellite Surface Materials," J. App. Phys., Vol. 43, No. 4, 1972, pp.1563-1572. 487.

${ }^{16}$ Krolikowski, W.F., and Spicer, W.E., "Photoemission Studies of the Noble Metals: II. Gold," Phys. Rev. B, Vol. 1, No. 2, 1970, pp. 478-

${ }^{17}$ Thomson, C.D., "Measurements of the Secondary Electron Emission Properties of Insulators," Ph.D. Dissertation; Physics Dept., Utah State University, Logan, UT, 2004.

${ }^{18}$ Hoffmann, Ryan, Abbot, Jonathan, and Dennison, JR, "Critical Electron Dose Threshold for Measuring the Electron Yield of Unbiased Highly Insulating Materials," American Physical Society March Meeting 2006, Baltimore, MD, Bull. Am. Phys. Soc. Vol. 51, No. 1 Part II, March 13-17, 2006.

${ }^{19}$ Nickles, Neal, Davies, R.E. and Dennison, J.R., "Applications of Secondary Electron Energy- and Angular-Distributions to Spacecraft Charging," Proceedings of the 6th Spacecraft Charging Technology Conference, Air Force Research Laboratory Science Center, Hanscom Air Force Base, MA, 2000.

${ }^{20}$ Dennison, J.R., Thomson, C. D., and Sim, Alec, "The effect of low energy electron and UV/VIS radiation aging on the electron emission properties and breakdown of thin-film dielectrics," Proceedings of the $8^{\text {th }}$ IEEE Dielectrics and Electrical Insulation Society (DEIS) International Conference on Solid Dielectrics (ICSD), IEEE, Piscataway, NJ, 2004, 967-971.

${ }^{21}$ Dennison, J.R., Sim, Alec, and Thomson, C. D., "Evolution of the Electron Yield Curves of Insulators as a Function of Impinging Electron Fluence and Energy," IEEE Transaction on Plasma Science, Vol. 34, No. 5, October, 2006, pp. 2204-2218.

${ }^{22}$ Olsen R.C., "The hidden population of the magnetosphere," J. Geophys. Res., Vol. 87, 1982, 3481-3488.

${ }^{23}$ Purvis, C.K. H.B. Garrett, A.C. Whittlesey, and N.J. Stevens, Design Guidelines for Assessing and Controlling Spacecraft Charging Effects, NASA TP 2361, 1984

${ }^{24}$ S. T. Lai and D. Della-Rose, "Spacecraft charging at geosynchronous altitudes: New evidence of the existence of critical temperature," $J$. Spacecr. Rockets, vol. 38, no. 6, 2001, pp. 922-928.

${ }^{24}$ Heroux, L.J. and Hinteregger, H.E., "Solar Ultraviolet Irrandiance," in Handbook of Geophysics and the Space Environment, Ed. Jursa, A.S., Air Force Geophysics Laboratory, National Technical Information Service, Springfield, VA, 1985, pp. 2-1 to 2-21 and sources therein.

${ }^{25}$ Lai, S.T. and Tautz, M., "Aspects of Spacecraft Charging in Sunlight," IEEE Transaction on Plasma Science, Vol. 34, No. 5, October, 2006, pp. 2053-2061.

${ }^{26}$ Lai, S.T., Gussenhoven, M.S., and Cohen, H.A., "The Concepts of Critical Temperature and Energy Cutoff of Ambient Electrons in High Voltage Charging of Spacecraft," in Proceedings of the 17th ESLAB Symposium, edited by D.Guyenne and J.H. A. Pedersen, European Space Agency, Noordwijk, The Netherlands, 1983, pp. 169-175.

${ }^{27}$ Lai, S.T., "Spacecraft Charging Thresholds in Single and Double Maxwellian Space Environments," IEEE Trans. Nucl. Sci., Vol.19, 1991, pp. 1629-1634.

${ }^{28}$ Laframboise, J.G., and Kamitsuma, M., "The Threshold Temperature Effect in High Voltage Spacecraft Charging," in Proceedings of Air Force Geophysics Workshop on Natural Charging of Large Space structures in Near Earth Polar Orbit, AFRL-TR-83-0046, ADA-134-894, 1983, pp. 293-308.

${ }^{29}$ Laframboise, J.G., Kamitsuma, M. and Goddard, R., "Multiple floating potentials, 'Threshold-temperature' effects and 'Barrier' effects”, in Proc. Int. Symp. On Spacecraft Materials in Space Env., Toulouse, France, 1982.

${ }^{30}$ Olsen, R.C., “A Threshold Effect for Spacecraft Charging,” J. Geophys. Res., Vol. 88, 1983, pp. 493-499. 
${ }^{31}$ Rubin, A.G., P.L. Rothwell and G.K. Yates, "Reduction of spacecraft charging using highly emissive surface materials, in Proceedings of the 1978 Symposium on the Effects of the Ionosphere on Space and Terrestrial Systems, ed. J.H. Goodman, Naval Research Laboratory, Washington, D.C. 1978, pp. 313-316.

32 Tobiaka, W., "SOLAR2000 v2.30 and SOLARFLARE v1.01: New Capabilities for Space System Operations," AIAA-2007-495, Proceedings of the 45 ${ }^{\text {th }}$ AIAA Meeting on Aerospace Sciences, Reno, NV, January 10, 2007.

${ }^{33}$ Minow, J. Dick,ann, Anne M., and Blackwell, William C., "Status of the L2 and Lunar Charged Particle Environment Models," AIAA2007-0910, Proceedings of the 45 th AIAA Meeting on Aerospace Sciences, Reno, NV, January 10, 2007.

34 European Space Agency (ESA), "Space Environment Information System (SPENVIS)," URL: http://www.spenvis.oma.be/spenvis/intro.html. [cited January 10, 2007].

${ }^{35}$ M. Tautz and S. T. Lai, "Analytic models for a rapidly spinning spherical satellite charging in sunlight," J. Geophys. Res., Vol. 110, No. A7, 2005, p. A07220.

${ }^{36}$ Reimer, L. Scanning Electron Microscopy, Springer-Verlag, Berlin, 1985.

${ }^{37}$ S. T. Lai, "High-level spacecraft charging at geosynchronous altitudes: A statistical study," in Proc. 8th Spacecraft Charging Technology Conf., 2003. NASA/CP-2004-213091.

${ }^{38}$ S. T. Lai and M. Tautz, "High-level spacecraft charging in eclipse at geosynchronous altitudes: A statistical study," J. Geophys. Res., Vol. 111, No. A9, 2005, p. A09201.

${ }^{39}$ M. Soop, "Numerical calculations of the perturbation of an electric field around a spacecraft," in Photons and Particle Interactions With Surfaces in Space, R. J. L. Grard, Ed. Dordrecht, Holland: Reidel, 1972.

${ }^{40}$ D. Higgins, "An analytic model of multi-dimensional spacecraft charging fields and potentials," IEEE Trans. Nucl. Sci., Vol. NS-26, No. 6, Dec. 1979, p. 5162.

${ }^{41}$ A. L. Besse and A. G. Rubin, "A simple analysis of spacecraft charging involving blocked photo-electron currents," J. Geophys. Res., Vol. 85, No. A5, May 1980, pp. 2324-2328.

${ }^{42}$ Dennison, J.R., Swaminathan, Prasanna, Jost, Randy, Brunson, Jerilyn, Green, Nelson, and Frederickson, A. Robb, "Improved Methods and Analysis for Resistivity Measurements Related to Spacecraft Charging," IEEE Transaction on Plasma Science, Vol. 34, No.5, October 2006, pp. 2191-2203.

${ }^{43}$ Dennison, J.R., Kite, Jason, Chang, W.Y., and Davies, R.E., “Absolute and Differential Spacecraft Charging as a Result of Evolving Surface Contamination," Proceedings of the $7^{\text {th }}$ Spacecraft Charging Technology Conference, Noordwijk, The Netherlands, 23-27 April 2000.

${ }^{44}$ Joy, D. C., “A Database on Electron-Solid Interactions," Scanning, Vol. 17; No. 5, 1995, pages 270.

${ }^{45}$ Clerc, Sébastien, Dennison, J.R., Hoffman, Ryan, and Abbott, Jonathan, "On the Computation of Secondary Electron Emission Models," IEEE Transaction on Plasma Science, Vol. 34, No.5, October 2006, pp. 2219-2225.

${ }^{46}$ Thomson, C.D., Dennison, J.R., Davies, R.E., Ferguson, D.C., Galafaro, J.T., and Vayner, B.V., "Investigation of the First Snapover of Positively Biased Conductors in a Plasma," Proceedings of the $38^{\text {th }}$ AIAA Meeting on Aerospace Sciences, Reno, NV, January 10 , 2000.

${ }^{47}$ Corbridge, J., Dennison, J.R., Hodges, J. Hoffmann, R.C., Abbott, J., Hunt, A., and Spaulding, R., "Measurements of the Radiation Induced Conductivity of Insulating Polymeric Materials for the James Webb Space Telescope," American Physical Society Four Corner Section Meeting, Utah State University, Logan, UT, October 6-7, 2006.

48 Davies, R.E., and J.R. Dennison, Evolution of secondary electron emission characteristics of spacecraft surfaces, J. Spacecraft and Rockets, Vol. 34, No. 4, 1997, pp. 571-574.

${ }^{49}$ Davies, R.E., An instrument for experimental secondary electron emission investigations, with application to the spacecraft charging problem, M.S. Thesis, Dept. of Physics, Utah State Univ., Logan, UT, 1996.

${ }^{50}$ Sternglass, E.J., "Backscattering of kilovolt electrons from solids," Phys. Rev., Vol., 95, 1954, pp. 345-358.

${ }^{51}$ Sternglass, E.J., "Theory of secondary electron emission by high-speed ions," Phys. Rev. Vol. 108, 1957, pp. 1-12.

${ }^{52}$ Young, J.R. "Penetration of Electrons and Ions in Aluminum," J. Appl. Phys., Vol. 27, 1956, p. 1; "Penetration of Electrons in Aluminum Oxide Films," Phys. Rev. Vol. 103, 1956, 292.

${ }^{53}$ Feldman, C., "Range of 1-10 keV electrons in solids," Phys. Rev. Vol. 117, 1960, 455-459.

${ }^{54}$ Schwarz, S.A., "Application of a semi-empirical sputtering model to SEE”, J. Appl. Phys., 1990.

${ }^{55}$ Dale Ferguson, NASA Marshal Space Fight Center, private communications, January, 2007.

${ }^{56}$ Dennison, J.R., Frederickson, A. Robb, Green, Nelson W., Benson, C.E., Brunson, Jerilyn and Swaminanthan, Prasanna, "Materials Database of Resistivities of Spacecraft Materials.," Charge Collector Database [online database], NASA Space Environments and Effects Program, NASA Marshal Space Flight Center, October, 2005. URL: http://see.msfc.nasa.gov/ee/db chargecollector.htm. [cited January 10, 2007].

${ }^{57}$ Grais, K. I., and A. M. Bastawros, "A study of secondary electron emission in insulators and semiconductors,” J. Appl. Phys. Vol. 53, 1982, pp. 5239-5242.

${ }^{58}$ Corbridge, Jodie, Dennison, J.R., and Nickles, Neal, "Secondary Electron Emission of Annealed Graphitic Amorphous Carbon: The Role of Evolving Bandgap,” American Physical Society March Meeting 2004, Montreal, Quebec, Canada, Bull. Am. Phys. Soc. Vol. 49, No. 1 Part II, March 22-26, 2004.

${ }^{59}$ Dennison, J. R., Holtz, M., and Swain, G., "Raman Spectroscopy of Carbon Materials," Spectroscopy, Vol. 11, No. 8, 1996, pp. 38-45.

${ }^{60}$ Stoner, J. O., “Accurate determination of carbon-foil surface densities,” J. Appl. Phys., Vol. 40, 1969, pp. 707-709. 